Korean J. Math. 22 (2014), No. 4, pp. 693-698

http://dx.doi.org/10.11568/kjm.2014.22.4.693

\title{
A STUDY ON THE CATEGORY OF NORMAL FUZZY HYPERGROUPS
}

\author{
IG SUng KIM
}

\begin{abstract}
Although the category NFHG of normal fuzzy hypergroups is not a topos, it forms a pseudo topos. Also we show that there are pseudo power objects in $N F H G$.
\end{abstract}

\section{Introduction}

Sun [3] showed that the category NFHG of normal fuzzy hypergroups satisfies all the axiom of topos except for the subobject classifier axiom. So we define a pseudo subobject classifier, pseudo topos and pseudo power object. Also Goldblatt [1] showed that any topos has power objects.

In this paper, we show that $N F H G$ has a pseudo subobject classifier. So $N F H G$ forms a pseudo topos. Also we show that there are pseudo power objects in NFHG which is not a topos.

\section{Preliminaries}

In this section, we state some definitions and properties which will serve as the basic tools for the arguments used to prove our results.

Received October 7, 2014. Revised December 11, 2014. Accepted December 11, 2014.

2010 Mathematics Subject Classification: 18B25.

Key words and phrases: normal fuzzy hypergroups, topos, pseudo power objects, pseudo topos.

This research was supported by Sangji University Research Fund, 2014.

(c) The Kangwon-Kyungki Mathematical Society, 2014.

This is an Open Access article distributed under the terms of the Creative commons Attribution Non-Commercial License (http://creativecommons.org/licenses/by -nc/3.0/) which permits unrestricted non-commercial use, distribution and reproduction in any medium, provided the original work is properly cited. 
Definition 2.1. An elementary topos is a category $\mathcal{E}$ that satisfies the following;

(T1) $\mathcal{E}$ is finitely complete,

(T2) $\mathcal{E}$ has exponentiation,

(T3) $\mathcal{E}$ has a subobject classifier.

(T2) means that for every object $A$ in $\mathcal{E}$, the endofunctor $(-) \times A$ has its right adjoint $(-)^{A}$. Hence for every object $A$ in $\mathcal{E}$, there exists an object $B^{A}$, and a morphism $e v_{A}: B^{A} \times A \rightarrow B$, called the evaluation map of $A$, such that for any $Y$ and $f: Y \times A \rightarrow B$ in $\mathcal{E}$, there exists a unique morphism $g$ such that $e v_{A} \circ(g \times i d)=f$;

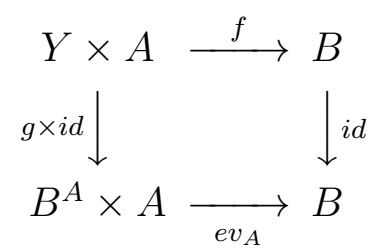

And subobject classifier in (T3) is an $\mathcal{E}$-object $\Omega$, together with a morphism $\top: \mathbf{1} \rightarrow \Omega$ such that for any monomorphism $h: D \rightarrow C$, there is a unique morphism $\chi_{h}: C \rightarrow \Omega$, called the character of $h: D \rightarrow C$ which makes the following diagram a pull-back;

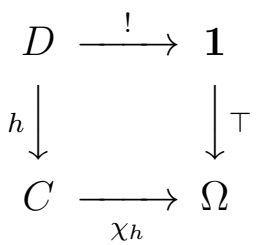

ExAmple 2.2. Category Set is a topos. $\{*\}$ is a terminal object. $\Omega=\{0,1\}$ and $\top:\{*\} \rightarrow \Omega$ with $\top(*)=1$ is a subobject classifier. If we define

$\chi_{h}=1$ if $c=h(d)$ for some $d \in D$,

$\chi_{h}=0$ otherwise

then $\chi_{h}$ is a characteristic function of $D$.

Let $H$ be a nonempty set and $F(H)=[0,1]^{H}$ be the set of all fuzzy subset of $H$ and $F^{*}(H)=F(H)-\{\phi\}$. A fuzzy hyperoperation on $H$ is a mapping $\star: H^{2} \rightarrow F(H)$ and the couple $(H, \star)$ is called a partial fuzzy hypergroupoid. If the fuzzy hyperoperation $\star$ maps $H^{2}$ into $F^{*}(H)$, then $(H, \star)$ is called a fuzzy hypergroupoid. 


\section{DEFINITION 2.3.}

(1) A fuzzy semihypergroup is a a fuzzy hypergroupoid $(H, \star)$ which satisfies the associative law.

(2) A fuzzy quasihypergroup is a a fuzzy hypergroupoid $(H, \star)$ which satisfies the reproductive law.

(3) A fuzzy hypergroup is a fuzzy semihypergroup which is also a fuzzy quasihypergroup

(4) A fuzzy subhypergroup $(A, \bullet)$ of a fuzzy hypergroup $(B, \bullet)$ is a nonempty subset $A \subseteq B$ such that for any $a \in A, a \bullet A=A=A \bullet a$.

Definition 2.4. A fuzzy hypergroup $(H, \star)$ is said to be normal if it satisfies the following three conditions;

(1) $(x \star x)(x)=1$ for all $x \in H$;

(2) $x \star y=x \star x \cup y \star y$ for all $x, y \in H$;

(3) $(x \star x)(z) \geq(x \star x)(y) \wedge(y \star y)(z)$ for all $x, y, z \in H$.

Let $N F H G$ be a category, where objects are normal fuzzy hypergroups and a morphism from $(H, \diamond)$ to $(K, \star)$ is a mapping $f: H \rightarrow K$ such that $f(a \diamond b) \subseteq f(a) \star f(b)$.

Definition 2.5. A pseudo subobject classifier in a category $\mathcal{E}$ is an object $\Omega$, together with a morphism $\top: \mathbf{1} \rightarrow \Omega$ such that for any $(A, \star) \subseteq(B, \star)$ and any inclusion $k: A \rightarrow B$, there is a unique morphism $\chi_{k}: B \rightarrow \Omega$ which makes the following diagram a pull-back;

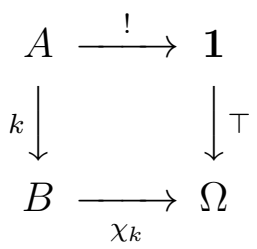

Definition 2.6. A pseudo topos is a category $\mathcal{E}$ that satisfies the following;

(T1) $\mathcal{E}$ is finitely complete,

(T2) $\mathcal{E}$ has exponentiation,

(T3) $\mathcal{E}$ has a pseudo subobject classifier.

Definition 2.7. A category $\mathcal{E}$ is said to have pseudo power objects if to each object $A$, there are objects $P(A)$ and $E(A)$, and inclusion $e: E(A) \rightarrow P(A) \times A$, such that for any object $B$, and "relation", 
$r: R \rightarrow B \times A$ there is exactly one morphism $f_{r}: B \rightarrow P(A)$ for which there is a pullback of the form

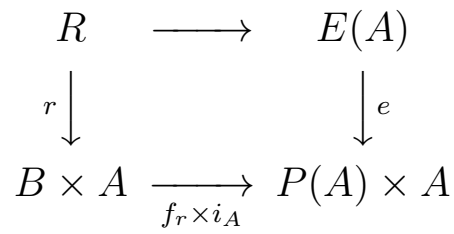

\section{Pseudo Topos NFHG and Pseudo Power Object}

TheOREM 3.1. NFHG has a pseudo subobject classifier.

Proof. Let $\Omega=\{\top, \perp\}$ and $\diamond: \Omega \times \Omega \rightarrow[0,1]^{\Omega}$ defined by

$(T \diamond \top)(T)=1=(T \diamond \top)(\perp)$,

$(\perp \diamond \perp)(T)=1=(\perp \diamond \perp)(\perp)$

$(\top \diamond \perp)=(\top \diamond \top) \cup(\perp \diamond \perp)$.

Then $(\Omega, \diamond)$ is a normal fuzzy hypergroup.

For any normal fuzzy subhypergroup $(K, \star) \subseteq(H, \star)$ and inclusion $f: K \rightarrow H$ defined by $f(k)=k$ for any $k \in K$, we construct a morphism $\chi_{f}: H \rightarrow \Omega$ defined by

$\chi_{f}(h)=\top$ if $x \in K$

$\chi_{f}(h)=\perp$ otherwise.

For any $z \in \Omega, \chi_{f}(u \star v)(z) \leq\left(\chi_{f}(u) \diamond \chi_{f}(v)\right)(z)=1$. So $\chi_{f}(u \star v) \subseteq$ $\chi_{f}(u) \diamond \chi_{f}(v)$. Thus $\chi_{f}: H \rightarrow \Omega$ is a morphism. For any $h:(M, \oplus) \rightarrow$ $(H, \star)$ and ! : $(M, \oplus) \rightarrow(\{*\}, \odot)$ with $\chi_{f} \circ h=\top \circ$ !, we have that $\chi_{f} \circ h=\top \circ !$ implies $h(m) \in \operatorname{Im}(f)$. That is, $h(m)=f(k)$ for some $k \in K$. So there exists a morphism $g:(M, \oplus) \rightarrow(K, \star)$ such that $g(m)=k$ with $h(m)=f(k)$ for all $m \in M$. Clearly, $f \circ g=h$ and such a morphism is unique.

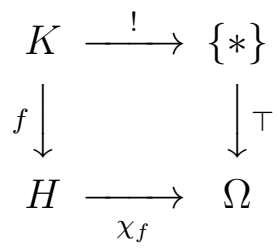

Corollary 3.2. NFHG is a pseudo topos. 
ThEOREM 3.3. In category $N F H G$, for each object $(A, \oslash)$ there are objects $(P(A), \star),(E(A), \triangle)$ and inclusion $g:(E(A), \triangle) \rightarrow(P(A), \star) \times$ $(A, \oslash)$ such that for any object $(B, \oplus)$ and relation $(R, \nabla)$ from $(A, \oslash)$ to $(B, \oplus)$, there is exactly one morphism $f_{r}:(B, \oplus) \rightarrow(P(A), \star)$ for which there is a pullback of the form

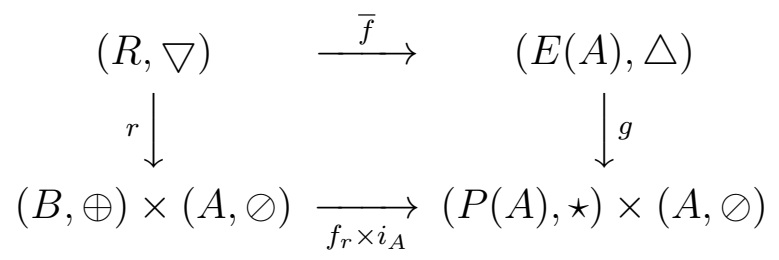

where $\left(\left(b_{1}, a_{1}\right) \nabla\left(b_{2}, a_{2}\right)\right)\left(r_{1}, r_{2}\right)=\left(\left(b_{1} \oplus b_{1}\right)\left(r_{1}\right) \wedge\left(a_{1} \oslash a_{1}\right)\left(r_{2}\right)\right) \vee\left(\left(b_{2} \oplus\right.\right.$ $\left.\left.b_{2}\right)\left(r_{1}\right) \wedge\left(a_{2} \oslash a_{2}\right)\left(r_{2}\right)\right)$ and $r(b, a)=(b, a)$.

Proof. Let $P(A)=(\Omega, \diamond)^{(A, \oslash)}=\{f: A \rightarrow \Omega\}$ where $\star: P(A) \times$ $P(A) \rightarrow[0,1]^{P(A)}$ defined by $(f \star f)(h)=\wedge(f(x) \diamond f(x)) h(x)$ and $E(A)=$ $\{<f, a\rangle \mid f \in P(A), a \in A, f(a)=\top\}$ where $\triangle: E(A) \times E(A) \rightarrow$ $[0,1]^{E(A)}$ defined by $((f, a) \triangle(g, b))(h, c)=((f \star f)(h) \wedge(a \oslash a)(c)) \vee$ $((g \star g)(h) \wedge(b \oslash b)(c))$. Then we obtain objects $(P(A), \star)$ and $(E(A), \triangle)$. Consider

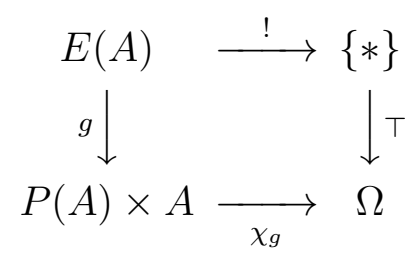

Let $\chi_{g}\langle f, a\rangle=f(a)$, then $\chi_{g}$ is a morphism and $\chi_{g} \circ g=$ T०!. By the property of $(P(A), \star)$ and $(E(A), \triangle), \Omega$ is a pseudo subobject classifier of the inclusion $g: E(A) \rightarrow P(A) \times A$. So the previous square is a pullback.

Consider

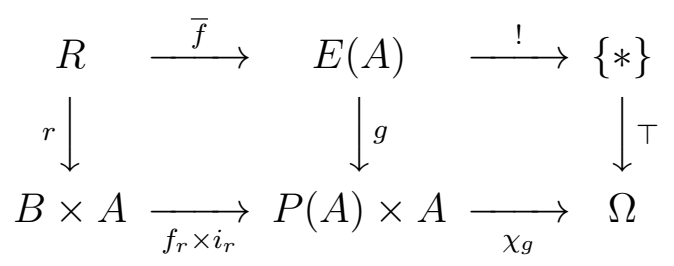

Let $f_{r}: B \rightarrow P(A)$ defined by

$\left(f_{r}(b)\right)(a)=(\top \circ !)<b, a>$, if $<b, a>\in R$

$\left(f_{r}(b)\right)(a)=\perp$, otherwise 
Then $f_{r}: B \rightarrow P(A)$ is a morphism. And $\Omega$ is a pseudo subobject classifier of the inclusion $r: R \rightarrow B \times A$ with $!: R \rightarrow\{*\}$. So the outer square is a pullback. By definition of pullback, there is exactly one morphism $\bar{f}: R \rightarrow E(A)$ such that $g \circ \bar{f}=\left(f_{r} \times i_{r}\right) \circ r$. By pullback Lemma, the left square is a pullback.

Corollary 3.4. NFHG has pseudo power objects.

\section{References}

[1] R. Goldblatt, Topoi, North-Holland, 1984.

[2] P. T. Johnstone, Topos Theory, Academic Press, N. Y., 1977.

[3] K. Sun, X. H. Yuan and H, Li, Fuzzy hypergroups based on fuzzy relation, Comput. Math. Appl (2010).

[4] X. H. Yuan, Comparison between Category Fuz and Category Set, J. of Fuzzy Math. 5 (5) (1997).

Ig Sung Kim

Department of Data Information

Sangji University

Wonju 220-702, Korea.

E-mail: iskim@sangji.ac.kr 